\title{
Epidemiology of Theileria bicornis among black and white rhinoceros metapopulation in Kenya
}

\author{
Moses Y Otiende ${ }^{1 *}$, Mary W Kivata ${ }^{4}$, Joseph N Makumi ${ }^{4}$, Mathew N Mutinda ${ }^{1}$, Daniel Okun ${ }^{4}$, Linus Kariuki ${ }^{1}$, \\ Vincent Obanda ${ }^{1}$, Francis Gakuya ${ }^{1}$, Dominic Mijele ${ }^{1}$, Ramón C Soriguer ${ }^{2}$ and Samer Alasaad ${ }^{2,3^{*}}$
}

\begin{abstract}
Background: A huge effort in rhinoceros conservation has focused on poaching and habitat loss as factors leading to the dramatic declines in the endangered eastern black rhinoceros (Diceros bicornis michaeli) and the southern white rhinoceros (Ceratotherium simum simum). Nevertheless, the role disease and parasite infections play in the mortality of protected populations has largely received limited attention. Infections with piroplasmosis caused by Babesia bicornis and Theileria bicornis has been shown to be fatal especially in small and isolated populations in Tanzania and South Africa. However, the occurrence and epidemiology of these parasites in Kenyan rhinoceros is not known.
\end{abstract}

Results: Utilizing $18 \mathrm{~S}$ rRNA gene as genetic marker to detect rhinoceros infection with Babesia and Theileria, we examined blood samples collected from seven rhinoceros populations consisting of 114 individuals of black and white rhinoceros. The goal was to determine the prevalence in Kenyan populations, and to assess the association of Babesia and Theileria infection with host species, age, sex, location, season and population mix (only black rhinoceros comparing to black and white rhinoceros populations). We did not detect any infection with Babesia in the sequenced samples, while the prevalence of T. bicornis in the Kenyan rhinoceros population was $49.12 \%$ (56/114). White rhinoceros had significantly higher prevalence of infection (66\%) compared to black rhinoceros (43\%). The infection of rhinoceros with Theileria was not associated with animal age, sex or location. The risk of infection with Theileria was not higher in mixed species populations compared to populations of pure black rhinoceros.

Conclusion: In the rhinoceros studied, we did not detect the presence of Babesia bicornis, while Theileria bicornis was found to have a $49.12 \%$ prevalence with white rhinoceros showing a higher prevalence (66\%) comparing with black rhinoceros (43\%). Other factors such as age, sex, location, and population mix were not found to play a significant role.

Keywords: Ixodid, Ticks, Piroplasms, Diceros bicornis michaeli, Ceratotherium simum simum

\section{Background}

The populations and distribution ranges of the black rhinoceros (Diceros bicornis) and the white rhinoceros (Ceratotherium simum) have declined in the whole of Africa. The rate of their population decline is faster than any other large terrestrial mammal in recent times [1], a fact that supports their endangered status and calls for robust international efforts towards their recovery. These rhinoceros have been exterminated in the

\footnotetext{
* Correspondence: motiende@kws.go.ke; sameralasaad@hotmail.com ${ }^{1}$ Veterinary Services Department, Forensic and Genetics Laboratory Kenya Wildlife Service, P.O Box 40241-00100, Nairobi, Kenya

EEstación Biológica de Doñana, Consejo Superior de Investigaciones Científicas (CSIC), Avda. Américo Vespucio s/n 41092, Sevilla, Spain Full list of author information is available at the end of the article
}

majority of African countries, while their range among the remaining principal countries; Kenya, Tanzania, Namibia, Zimbabwe and South Africa [1] is greatly reduced and currently restricted in artificially created sanctuaries. Habitat loss and vicious poaching are the leading twin drivers of population decline of the rhinoceros [2]. However infectious diseases are also an incipient threat to endangered species [3] having been classified among the top five causes of species extinctions [4].

Piroplasms, which are blood-borne protozoan parasites in the genera Babesia and Theileria (Order Piroplasmidae), are globally distributed and transmitted by a diverse species of Ixodid ticks. These parasites infect a wide range of domesticated and wild mammals as well as humans. Infections may lead to severe disease and death or it may 
remain latent depending on virulence of the species and host immune status. Piroplasms have historically been known to infect rhinoceros with some infections associated with fatalities [5-7]. However the causal species were unknown until 10 years ago when Babesia bicornis and Theileria bicornis were independently associated with stress-induced mortality [8]. The first genetic work on piroplasms by Otiende et al., [9] has shown the existence of infection by piroplasm and the occurrence of three new haplotypes of Theileria bicornis circulating in both black and white rhinoceros in Kenya. The factors influencing piroplasms prevalence among Kenyan populations were previously unknown. Piroplasms have coevolved with rhinoceros and they coexist with the host without signs of clinical disease. However, stress induced by translocation has been linked to immune suppression and is a major cause of post translocation morbidity and/or fatality. Translocation is at the core of in situ management of rhinoceros metapopulation and yet it is incriminated as a disease inducer besides its inherent role in the spread of pathogens. The link between translocation and piroplasmosis is intricate because it is based on the modulatory effects of stress hormones on the immune system. Translocation elicits stress hormones, which allow uninhibited proliferation of piroplasms in the host resulting in disease and death. However, effects of stress hormones are not predictable or homogenous in the population as underlying individuals' conditions, such as injury, pregnancy, co-infection, vary and may elicit different immune response [10].

The goal of this study is to determine the epidemiology of Theileria biconis in Kenya. Specifically, we intended to (a) determine their prevalence in both species of rhinoceros and among sub-populations then (b) test the association of infection prevalence with host species, age, sex, location, season and population mix (black rhinoceros vs black and white rhinoceros populations). Information generated will be useful in guiding management and veterinary options such as translocation, differential diagnosis and chemotherapy.

\section{Methods}

\section{Study area}

Lake Nakuru National Park (LNNP) central coordinates are $0^{\circ} 22^{\prime} \mathrm{S} 36^{\circ} 05^{\prime} \mathrm{E}$ and is $4 \mathrm{~km}$ from Nakuru town center. The park covers an area of $188 \mathrm{~km}^{2}$ completely fenced, of which $44 \mathrm{~km}^{2}$ lies in the shallow alkaline soda lake, thereby leaving $144 \mathrm{~km}^{2}$ for wildlife use. The area around the lake is flat bare lowland of $1200 \mathrm{~m}$ altitude surrounded by hills and gentle cliffs that rise to $1750 \mathrm{~m}$ above sea level. The park receives mean annual rainfall of $850 \mathrm{~mm}$ with rainfall in the months of April to May and again in October - November. The park consists of open grassland with elevated areas occupied by dry forests of Acacia xanthophloea, Olea capensis sub sp. Macrocarpa and Croton dichogamus. Marshland along the river inflows and springs are covered by Cyperus laevigatus and Typha spp. Other striking plant species include the invasive Tarchonanthus spp. bush land, the deciduous (Teclea $\mathcal{E}$ Olive) forest and the Euphorbia candelabrum forest. The park has 33 white and 69 black rhinoceros that freely interact with other diverse species.

Nairobi National Park (NNP) central coordinates are $1^{\circ} 16^{\prime} \mathrm{S}, 36^{\circ} 49^{\prime} \mathrm{E}$ and is about $10 \mathrm{~km}$ from the city of Nairobi and covers an area of $117 \mathrm{~km}^{2}$. A large section of the park is fenced with only $20 \mathrm{~km}$ left open for wildlife dispersal. Average annual rainfall is $800 \mathrm{~mm}$ with rainy season between April-May and October-November. The vegetation consists of mosaic grassland, thickets and Acacia and deciduous forests as well as woodlands especially along River Mbagathi that crosses it. The park has 77 black and 13 white rhinoceros besides many other wildlife species.

Ngulia rhino Sanctuary (NgRS) is within Tsavo West National Park occupying a fenced area of $90 \mathrm{~km}^{2}$ at $3^{0}$ $01^{\prime} \mathrm{S}$ to $3^{0} 06^{\prime} \mathrm{S}$ and $38^{\circ} 06 \mathrm{E}$ to $38^{\circ} 10^{\prime} \mathrm{E}$. Altitude ranges from $600 \mathrm{~m}$ of lowlands to $1800 \mathrm{~m}$ of craggy hills with average annual rainfall of $600 \mathrm{~mm}$. Dry period is between December to March, while rains occur in the months of April to June and again in October and November. The vegetation is thickly wooded by Commiphora-Acacia woodland, dotted with baobab trees. This sanctuary contains 77 black rhinoceros without white rhinoceros, though other small-medium sized wildlife occurs in small density.

Meru Rhino Sanctuary (MRS) is $48 \mathrm{Km}^{2}$ (central coordinates, $\mathrm{N} 00^{\circ} 15.125$, E $038^{\circ} 06.481$ ) located within the Meru National Park has 44 black and 45 white rhinoceros. Altitude ranges from 1000 to $3400 \mathrm{~m}$ above the sea level. Average rainfall is $635-762 \mathrm{~mm}$ with the wet season occurring in late March to April, while the dry season begins from October. Major rivers such as Makutano, Kanjoo, Kathithi, Rujuwero and Kindani traverse MRS, which contributes to mosaic vegetation types that include thickets, bushland and grassland as well as a thick forest on its southern edge.

Solio and Mugie Rhino Sanctuaries are in the LaikipiaSamburu ecosystem, which is characterized by savannahtype grassland dominated by Euclea divinorum, Acacia spp and Euphorbia woodland while annual rainfall averages 300 to $700 \mathrm{~mm}$. Rainfall level varies annually with intermittent patterns that peaks in April-May, July-August and October-November. The rest of the months are dry. Solio Sanctuary, $0^{\circ} 16^{\prime} \mathrm{S} 37^{\circ} 00^{\prime} \mathrm{E} / 0.27^{\circ} \mathrm{S} 37^{\circ} \mathrm{E} /-0.27$; 37 , is $68.9 \mathrm{~km}^{2}$ and has altitude of $1932 \mathrm{~m}$, located at the base of the Aberdare ranges. The sanctuary holds 50 black and 110 white rhinoceros. Mugie rhino sanctuary (ceased to be a rhino sanctuary at the time of this study, as the entire 
population was translocated) was $90 \mathrm{~km}^{2}$ with an altitude $1990 \mathrm{~m}$.

\section{Sampling design}

This study was carried out between 2011 and 2012 in various sub populations of black and white rhinoceros in Kenya. Sampling was cross-sectional whereby samples were collected from apparently healthy rhinos immobilized for translocation or tagging. The biodata of each rhinoceros was obtained from the KWS rhinoceros database. Rhinoceros $<2$ years of age were not immobilized for translocation. Juvenile rhinoceros are $<3.5$ years, sub-adults are (3.6 -7 years) and adults are $>7$ years. Rhinoceros were immobilized using a combination of

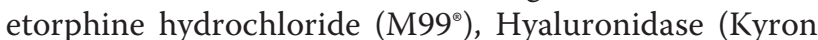
Laboratories, Benrose 2011, South Africa) and xylazine (Norvatis, [PTY] Ltd, South Africa). Venous blood was drawn from the front limb of the rhinoceros and then collected in EDTA tubes. Blood in EDTA tubes were gently mixed by turning the tubes up and down and then transferring aliquots in labeled cryovials followed by quick freezing in liquid nitrogen. The samples were transported in liquid nitrogen to Forensic and Genetics Laboratory of Kenya Wildlife Service in Nairobi for analysis.

\section{DNA isolation and PCR amplification}

Genomic DNA was extracted from blood using a genomic DNA extraction kit (DNeasy blood and Tissue Kit, QIAGEN, Southern Cross Biotechnologies, South Africa) following the manufacturers' protocol. A nested PCR amplification specific for the 18S rRNA gene of Babesia and Theileria was performed. A primary amplification was carried out in $50 \mu \mathrm{l}$ reaction containing $3 \mu \mathrm{l}$ of the genomic DNA, $45 \mu \mathrm{l}$ of Platinum blue supermix, $1 \mu \mathrm{l}$ $(10 \mathrm{mM})$ each forward and reverse primers. The forward primer was ILO-9029, (5'-CGGTAATTCCAGCTCCAA TAGCGT-3') and reverse, ILO-9030 (5'-TTTCTCTC AAAGGTGCTGAAGGAGT-3') primer [11]. The amplification (Thermocycler, Veriti, ABI) was preceded by a $30 \mathrm{sec}$ polymerase activation step at $95^{\circ} \mathrm{C}$ followed by 30 cycles of $1 \mathrm{~min}$ each at $94^{\circ} \mathrm{C}$, annealing at $53^{\circ} \mathrm{C}$ for $30 \mathrm{sec}$, extension for $1 \mathrm{~min}$ at $72^{\circ} \mathrm{C}$. Amplification was terminated by a final extension step $72^{\circ} \mathrm{C}$ for $9 \mathrm{~min}$. The secondary amplification was in a $50 \mu \mathrm{l}$ reaction containing $2 \mu \mathrm{l}$ of the primary amplification product, $45 \mu \mathrm{l}$ of Platinum blue supermix, $1.5 \mu \mathrm{l}(10 \mathrm{mM})$ each of forward and reverse primers. The forward primer was MWG4/70, (5'-AGCTCGTAGTTGAATTTCTGCTGC- ${ }^{\prime}$ ') and the reverse was ILO-7782 (5'-AACTGACGACCTCCAAT CTCTAGTC-3') [11]. The secondary PCR (Thermocycler, Veriti, ABI) was initiated with an initial denaturation at $95^{\circ} \mathrm{C}$ for $30 \mathrm{sec}$, followed by 30 cycles of $1 \mathrm{~min}$ each at $94^{\circ} \mathrm{C}$, annealing at $55^{\circ} \mathrm{C}$ for $30 \mathrm{~s}$ and extension at $72^{\circ} \mathrm{C}$ for 1 min. The PCR was completed with a final extension step of $72^{\circ} \mathrm{C}$ for $9 \mathrm{~min}$. PCR products showing successful amplification on agarose gel analysis were directly sequenced for both strands. PCR products were purified for direct sequencing by enzymatic treatment using exonuclease I and shrimp alkaline phosphatase (PCR Product Presequencing Kit, Amersham). All DNA sequencing was carried out by direct cycle sequencing on both strands of purified PCR DNA products from PCR amplification. Sequencing reactions were carried out with the ABI PRISM DigDye Terminator v3.1 cycle sequencing kit and analyzed on an ABI310 DNA sequencer (Applied Biosystems, CA).

\section{Statistical analysis}

Statistical analyses were performed using Fisher Exact test for count data and Chi Square test to determine the relationship between infection of rhinoceros with Theileria and the following variables: rhinoceros age, location, sex, and rhinoceros species. To confirm our results, we also used a Generalized Linear Model with a binomial error, and a complementary log-log link function. All possible interactions were included in the first model. As rhinoceros species are essentially clustered with locality (game park or sanctuary) a generalized linear mixed effect model was also applied, considering locality (game park or sanctuary) as random effect: Theileria Infection Rhinos Species $+(1 \mid$ Locality). Statistical significance was assessed at $p<0.05$.

\section{Ethic}

The Committee of the Department of Veterinary and Capture Services of the Kenya Wildlife Service (KWS) approved the study including animal capture, translocation and sample collection. KWS guidelines on Wildlife Veterinary Practice-2006 were followed. All KWS veterinarians were guided by the Veterinary Surgeons Act Cap 366 Laws of Kenya that regulates veterinary practice in Kenya.

\section{Results}

A total of 114 blood samples of black $(n=82)$ and white rhinoceros $(n=32)$ were sampled from seven rhinoceros populations and molecularly examined for infection with Babesia and Theileria. We did not detect any infection with Babesia in the obtained sequences, while the overall Theileria prevalence was $49.1 \%$ (56/114). All Theileria sequences belonged to the three haplotypes already described by Otiende et al., [9]. The prevalence of Theileria infection was higher in white rhinoceros (66\%) than in black rhinoceros (43\%) (Table 1). We confirmed this result using a generalized linear model $(b=-0.652$, $p=0.023)$. The simplified glm model was Theileria Infection $\sim$ Rhinos Species, family $=$ binomial $(\operatorname{cloglog})$ : 


\begin{tabular}{|c|c|c|c|c|c|}
\hline Variable & Variable categories & $\%$ Negative & \% Positive & $\mathbf{N}$ & p-value \\
\hline \multirow[t]{3}{*}{$\overline{\mathrm{AGE}}$} & Juvenile & 58.8 & 41.2 & 17 & 0.764 \\
\hline & Sub-Adult & 48.2 & 51.8 & 56 & \\
\hline & Adult & 51.2 & 48.8 & 41 & \\
\hline \multirow[t]{6}{*}{ LOCATION } & LNNP & 46.7 & 53.3 & 35 & 0.559 \\
\hline & Meru N. P. & 50.0 & 50.0 & 12 & \\
\hline & Mugie & 57.9 & 42.1 & 20 & \\
\hline & Ngulia & 55.2 & 44.8 & 29 & \\
\hline & NNP & 55.6 & 44.4 & 10 & \\
\hline & Solio & 87.5 & 12.5 & 8 & \\
\hline \multirow[t]{2}{*}{ SEX } & Female & 41.7 & 58.3 & 59 & 0.7026 \\
\hline & Male & 30.0 & 70.0 & 55 & \\
\hline \multirow[t]{2}{*}{ SPECIES } & C. simum & 34.4 & 65.6 & 32 & 0.037 \\
\hline & D. bicornis & 57.3 & 42.7 & 82 & \\
\hline
\end{tabular}

Theileria Infection $\sim 0.0656 \pm 0.2287$ Rhinos Species $0.6516 \pm 0.2857$. Since prevalence was higher in white rhinoceros, we tested whether presence of white rhinoceros together with black rhinoceros in the same locality was a risk factor for infection with Theileria in black rhinoceros. We found no significant association between infection of black rhinoceros with Theileria and the presence or absence of white rhinoceros in the same locality $\left(x^{2}=0321 ; p=0.571\right)$. The results were confirmed by applying a generalized lineal mixed effect model, considering locality (game park or sanctuary) as random effect. The last model in this case was Theileria Infection $\sim$ Rhinos Species, family $=$ binomial (logit): Theileria Infection $\sim 0.0647 \pm 0.3722-0.9414 \pm 0.434$ (Rhinos Species). Prevalence seemed to increase with age, but the infection by age-groups (juveniles, sub-adults and adults) was not statistically significant (Fisher test, $p=0.764$, Table 1). Females of both species had higher prevalence $54 \%(29 / 54)$ than males $45 \%(27 / 60)$, this difference however was not statistically significant (Fisher test, $p=0.702$, Table 1 ). Inter-population variations in prevalence (Figure 1) were not statistically different (Fisher test, $p=0.681$, Table 1 ).

\section{Discussion}

The six sampled rhinoceros sub-populations in Kenya were infected with piroplasms but we molecularly detected only Theileria and not Babesia in all studied samples from black and white rhinoceros species.

Ticks and wildlife are the maintenance hosts of piroplasms but efficient transmission fundamentally requires presence of the protozoan, a competent tick species and the host.
Even with B. bicornis and T. bicornis originally identified from black rhinoceros [8] T. bicornis has now been detected in white rhinoceros, Nyala (Tragelaphus angasii) and Cattle [12-14]. This means that T. bicornis is a multihost pathogen with possibility of having diverse tick species as vectors. Since $D$. rhinocerinus have been found in other animals such as cattle, sheep, donkey, elephant (Loxodonta africana), buffalo (Syncerus caffer) and eland (Taurotragus oryx) $[15,16]$ this tick could be important in the cross-transmission of rhinocerotid piroplams. Pathogenicity of $T$. bicornis remains unresolved but T. equi, which is its close relative $[8,17]$ and recently seen in white rhinoceros [13], has been reported to cause clinical piroplasmosis in translocated equids [18].

Translocation is intimately associated with flare up of latent infections that result in clinical state [18-20]. This is because translocation leads to elevation of glucocorticoids, whose effects are viewed to be obligatorily immunodepressant [10], yet in most cases, especially in transient acute stress, they prepare an animal to survive [10,21,22]. In the present study, sampling was carried out on asymptomatic individuals and despite underlying infection with T. bicornis, some of them were subjected to a longer period of stressor condition; during $>1000 \mathrm{~km}$ road transportation to a new sanctuary in Ruma National Park. Nevertheless, for six months post-release monitoring of this population, they remained asymptomatic. This outcome may support the notion that T. bicornis is apathogenic or it may suggest that translocation-stress did not suppress immunity to induce clinical state. Theories behind disease induction by translocation-stress often focus on single parasite infections. However, in nature, wild animals are infected and infested simultaneously with a 


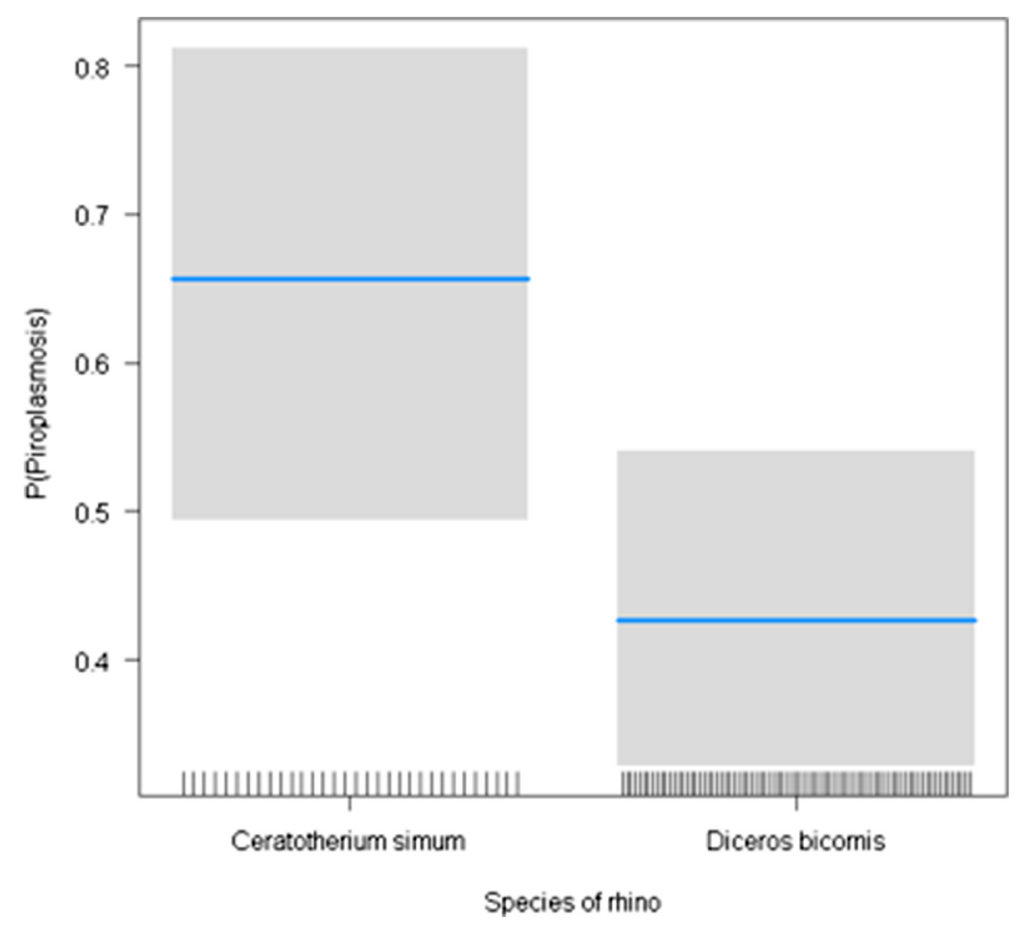

Figure 1 Variation of infection intensity of rhinoceros by species.

plethora of parasites that elicits complex immune response that may promote one parasite over the other. For instance, in concomitant infection involving African trypanosome superimposed with piroplasm leads to inhibition of the piroplasm in spite of the trypanosome immunodepressant effect [23]. In reference to fatal piroplasmosis [8], the deceased rhinoceros were subject to diverse and combined stressors; two black rhinoceros in Tanzania did not undergo prior capture and translocation event; the third fatal case involved high parasitemia, severe cold and injury while the fourth case was pregnant and developed translocation myopathy. This suggests that stressor factors that trigger clinical disease are many with maximum effect attained under synergistic state.

In the present study, the prevalence of $T$. bicornis was relatively high $(49.1 \%)$ but clinical disease was absent in the metapopulation, a state that could mimics endemic stability [24]. This state, which was initially coined for bovine babesiosis and now widely applied in many diseases and hosts, is based on the premise that (1) severity of clinical disease increases with age and (2) that after one infection, the probability that subsequent infections result in disease is reduced [25].

We noted that higher prevalence of $T$. bicornis (odds ratio, 2.502) being detected in white rhinoceros than in black species (Table 1) indicating a species effect. However, we did not find significant effect associating species with prevalence, suggesting that white rhinoceros, even though more susceptible, is not a risk factor to black rhinoceros prevalence. Our result show that the Kenyan white rhino has higher prevalence of $T$. bicornis (66\%) compared to $32.1 \%-46.6 \%$ in the South African populations $[13,26]$. The high prevalence of T. bicornis in white rhinoceros suggests they are important hosts in the epidemiology of this piroplasm. On the contrary, according to a theory postulated by Schmidt \& Ostfeld, [27] we suggest that white rhinoceros could benefit black rhinoceros by acting as 'sinks' for rhinocerotid piroplasms.

Further, our results show that prevalence among the agegroups of rhinoceros did not differ significantly (Table 1) contrary to the infection pattern in white rhinoceros population in South Africa in which female sub-adults had significantly higher prevalence [13]. Nevertheless, the ageassociated inclination in our result is comparable with that of Govender et al., [13] in that peak infections were observed among sub-adults (Table 1). It is postulated that sub-adult rhinoceros of both sexes are subject to numerous stress-related changes such as, reproductive maturity, courtship, mating and territorial fights $[13,28]$ that may suppress immunity and enhance susceptibility $[29,30]$.

Sex-biased prevalence is observed in many parasitic infections with males having higher prevalence and intensity of infections than their conspecific females [31]. In our results, there was no significant sex-biased difference in prevalence (Table 1) though females (56\%) seemed to have higher prevalence than males (45\%) an observation 
that concurs with that of Govender et al., [13]. Factors predisposing females to piroplasm are likely to be comparable with those affecting sub-adult rhinoceros.

The occurrence of $T$. bicornis in all the sampled rhinoceros sub-populations could have been facilitated by the regular translocations of individuals. Translocation assists spread of both tick vector and haemoparasites among habitat patches/populations. We observed apparent variations in prevalence among the sub-populations (Figure 2) but there was no significant difference (Table 1). This means that local factors in these habitats, such as ecological and weather differences, mammalian diversity, sanctuary size, were not sufficient to cause significant disparity in prevalence. According to Lopez et al., [32], frequent introduction of parasites in to a patch/habitat via host migration contributes to local patch prevalence. This implies that rhino sanctuaries' that frequently receive new individuals are likely to harbor higher parasite prevalence. Okita-Ouma et al., [33] points out that LNNP is more of a source population that has supported 41

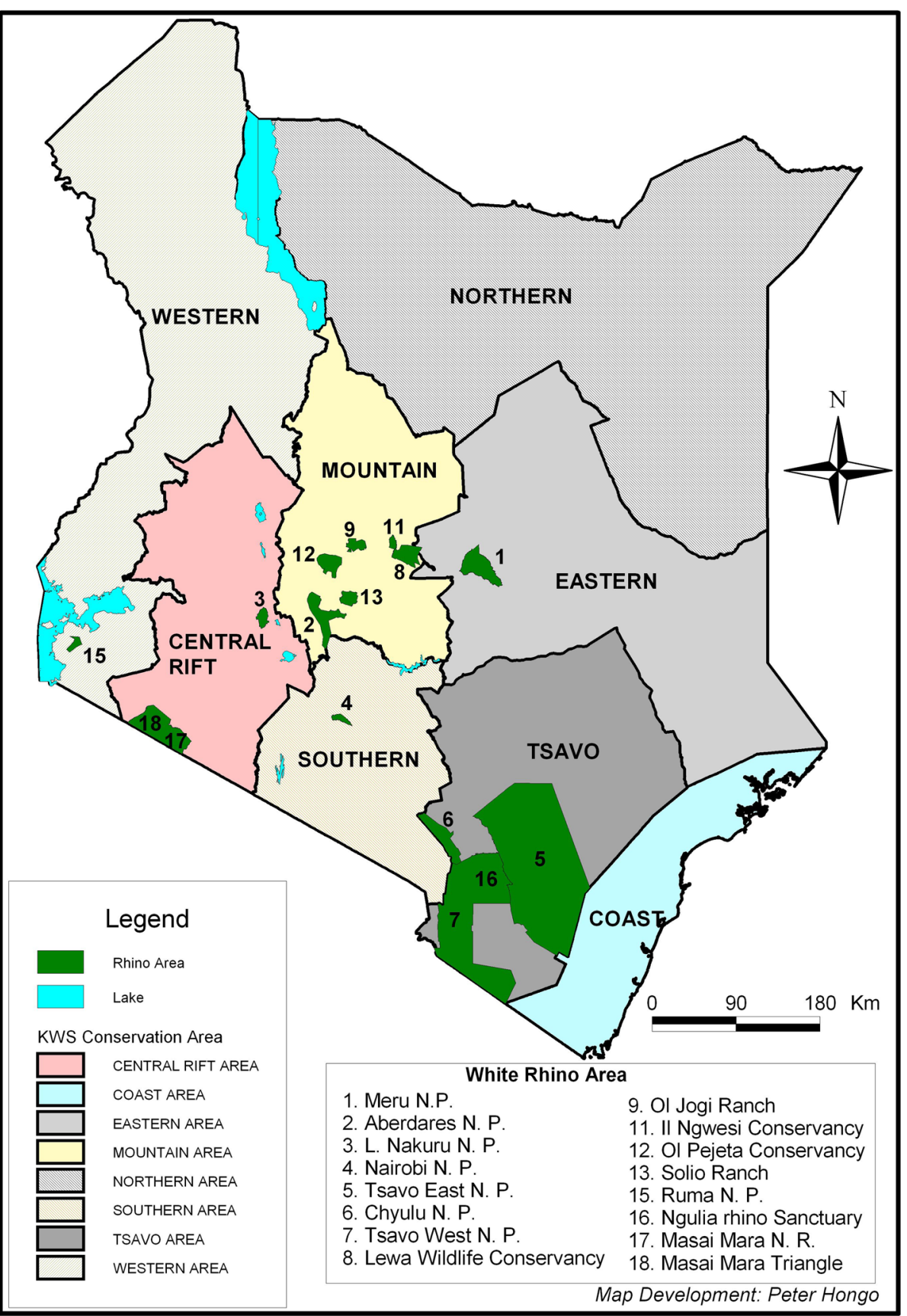

Figure $\mathbf{2}$ Locations of black rhinoceros conservation areas in Kenya. 
outward translocations and received one inward translocation, whereas Ngulia RS is a recipient population having received 16 inward translocations and only one outward translocation. However, lack of significant association between location and prevalence (Table 1) does not concur with the postulation of Lopez et al., [32].

\section{Conclusion}

In the analyzed samples we did not detect the presence of Babesia bicornis, while Theileria bicornis was found to have $49.12 \%$ prevalence with white rhinoceros showing a higher prevalence than black rhinoceros. Other factors such as age, sex, location, and population mix were not found to play a significant role.

\section{Competing interests}

The authors declare no conflict of interest in this work.

\section{Authors' contributions}

Conceived and designed the experiments: MYO SA VO. Performed the experiments: MWK MNM JNM. Analyzed the data DO LK FG DM RCS. Contributed reagents/materials/analysis tools: RCS SA. Wrote the paper: MYO SA. All authors read and approved the final manuscript.

\section{Author details \\ ${ }^{1}$ Veterinary Services Department, Forensic and Genetics Laboratory Kenya Wildlife Service, P.O Box 40241-00100, Nairobi, Kenya. ${ }^{2}$ Estación Biológica de Doñana, Consejo Superior de Investigaciones Científicas (CSIC), Avda. Américo Vespucio s/n 41092, Sevilla, Spain. ${ }^{3}$ Institute of Evolutionary Biology and Environmental Studies (IEU), University of Zürich, Winterthurerstrasse 190, 8057 Zürich, Switzerland. ${ }^{4}$ Department of Biochemistry and Biotechnology, Kenyatta University, P.O Box 43844-00100, Nairobi, Kenya.}

Received: 20 June 2014 Accepted: 24 December 2014

Published online: 17 January 2015

\section{References}

1. Kelly JD, Blyde DJ, Denney IS. The importation of the black rhinoceros (Diceros bicornis) from Zimbabwe into Australia. Aus Vet J. 1995;72:369-74.

2. Altizer S, Harvell D, Friedle E. Rapid evolutionary dynamics and disease threats to biodiversity. Trends Ecol Evol. 2003;589:596.

3. Daszak P, Cunningham AA, Hyatt AD. Emerging Threats to Infectious Diseases Wildlife- Health Biodiversity and infectious. Adv Sci. 2000;287:443-9.

4. Wilcove DS, Rothstein D, Dubow J, Phillips A, Losos E. Quantifying Threats to Imperiled Species in the United States. Bioscience. 1998;48:607-15.

5. MucCullogh B, Achard PL. Mortalities associated with capture, translocation, trade and exhibition of black rhinoceroses. Int Zoo. 1960;9:184-95

6. Brocklesby DW. A Babesia species of the black rhinoceros. Vet Rec. 1967:80:484

7. Mugera GM, Wandera JG. Degenerative polymyopathies in East African domestic and wild animals. Vet Rec. 1967;80:410-3.

8. Nijhof AM, Penzhorn BL, Lynen G, Mollel JO, Morkel P, Bekker CPJ, et al. Babesia bicornis sp. nov. and Theileria bicornis sp. nov.: tick-borne parasites associated with mortality in the black rhinoceros (Diceros bicornis). J Clin Microbiol. 2003;41:2249-54.

9. Otiende MY, Kivata MW, Jowers MJ, Makumi JN, Runo S, Obanda V, et al. Three Novel Haplotypes of Theileria bicornis in Black and White Rhinoceros in Kenya. Transbound Emerg Dis. 2014; [Epub ahead of print].

10. Martin LB. Stress and immunity in wild vertebrates: Timing is everything. Gen Com Endocr. 2009;163:70-6.

11. Maamun JM, Suleman MA, Akinyi M, Ozwara H, Kariuki T, Carlsson H-E. Prevalence of Babesia microti in free-ranging baboons and African green monkeys. J Parasitol. 2011;97:63-7.

12. Muhanguzi D, Matovu E, Waiswa C. Prevalence and characterization of Theileria and Babesia species in cattle under different husbandry systems in western Uganda. Int J Anim Veter Adv. 2010;2:51-8.
13. Govender D, Oosthuisen MC, Penzhorn BL. Piroplasm parasites of white rhinoceroses (Ceratotherium simum) in the Kruger National Park, and their relation to anaemia. J S Afr Vet Assoc. 2011;82:36-40.

14. Pfitzer S, Oosthuizen MC, Bosman AM, Vorster I, Penzhorn BL. Tick-borne blood parasites in nyala (Tragelaphus angasii, Gray 1849) from KwaZuluNatal, South Africa. Vet Parasitol. 2011;176:126-31.

15. Arthur DR. Ticks. A Monograph of the Ixodoidea. Part V. The Genera Dermacentor, Anocentor, Cosmiomma, Boophilus and Margaropus. London: Cambridge University Press; 1960

16. Keirans JE. Dermacentor rhinocerinus (Denny 1843) (Acari: Ixodida: Ixodidae): redescription of the male, female and nymph and first description of the larva. J Vet Res. 1993;60:59-68.

17. Katzer F, McKellar S, Kirvar E, Shiels B. Phylogenetic analysis of Theileria and Babesia equi in relation to the establishment of parasite populations within novel host species and the development of diagnostic tests. Mol Biochem Parasit. 1998:95:33-44.

18. Dennig HK. The isolation of Babesia species from wild animals. P ICP Rome. 1965;24-26:262-3.

19. Nijhof AM, Pillay V, Steyl J, Prozesky L, Stoltsz WH, Lawrence A, et al. Molecular Characterization of Theileria Species Associated with Mortality in Four Species of African Antelopes Molecular Characterization of Theileria Species Associated with Mortality in Four Species of African Antelopes. J Clin Microbiol. 2005;43:5907-11.

20. Obanda V, Kagira JM, Chege S, Okita-Ouma B, Gakuya F. Trypanosomosis and other co-infections in translocated black (Diceros bicornis michaeli) and white (Ceratotherium simum simum) rhinoceroses in Kenya. Sci Parasitol. 2011;12:103-7.

21. Wingfield JC, Maney DL, Breuner CW, Jacobs JD, Lynn S, Ramenofsky M, et al. Ecological bases of hormone-behavior interactions: the "emergency life history stage.". Am Zool. 1998;38:191-206.

22. Dhabhar FS. Stress-induced augmentation of immune function- The role of stress hormones, leukocyte trafficking, and cytokines. Brain Behav Immun. 2000;16:785-98.

23. Millott SM, Cox FEG. Interactions between Trypanosoma brucei and Babesia spp. and Plasmodium spp. in mice. Parasitology. 1985;90:241-54.

24. Penzhorn BL, Oosthuizen MC, Bosman A-M, Kilian JW, Horak IG. Black rhinoceros (Diceros bicornis) populations in northwestern Namibia are apparently not infected with piroplasms. J Wildl Dis. 2008;44:1032-5.

25. Coleman PG, Perry BD, Woolhouse MEJ. Endemic stability-a veterinary idea applied to human public health. Lancet. 2001;357:1284.

26. Bigalke RD, Keep ME, Keep PJ, Schoeman JH. A large Babesia sp. and a Theileria like piroplasm of the square-lipped rhinoceros. J S Afr Vet Assoc. 1970;41:292-4.

27. Schmidt K, Ostfeld R. Biodiversity and the dilution effect in disease ecology. Ecology. 2001;82:609-19.

28. Brett R. Mortality factors and breeding performance of translocated black rhinos in Kenya: 1984-1995. Pachyderm. 1998;69:82.

29. Glaser R, Kiecolt-Glaser JK. Stress-induced immune dysfunction: implications for health. Nat Rev Immunol. 2005;5:243-51.

30. Muehlenbein MP. Intestinal parasite infections and fecal steroid levels in wild chimpanzees. Am J Phys Anthropol. 2006;130:546-50.

31. Klein SL. Hormonal and Immunological mechanisms mediating sex differences in parasite infection. Parasit Immunol. 2004;26:247-64.

32. Lopez J, Gallinot LP, Wade MJ. Spread of parasites in metapopulations: An experimental study of the effects of host migration rate and local host population size. Parasitology. 2005;130:323-32.

33. Okita-Ouma B, Amin R, Van Langevelde F, Leader-Williams N. Density dependence and population dynamics of black rhinos (Diceros bicornis michaeli) in Kenya's rhino sanctuaries. Afr J Ecol. 2010:48:791-9. 